\title{
Análisis de la distribución espacial \\ de equipamientos educativos (0-16 años) en Extremadura a escala de detalle
}

Analysis of the spatial distribution of educational equipment ( $0-16$ years) in Extremadura in detail scale

\author{
Ana Nieto Masot \\ ananieto@unex.es \\ Área de Geografía Humana \\ Universidad de Extremadura (España) \\ Nieves Márquez Segovia \\ mmarquezte@alumnos.unex.es \\ Área de Geografía Humana \\ Universidad de Extremadura (España)
}

\section{Resumen}

La educación supone uno de los derechos universales más esenciales, por ello es necesario que se garantice su accesibilidad a toda la población y en especial a las zonas más desfavorecidas. Por ello, en este trabajo se estudiarán los recursos educativos en edades tempranas (Educación Infantil, Primaria y Educación Secundaria Obligatoria) en una región eminentemente rural y con baja densidad demográfica como es el caso de Extremadura para comprobar si existe una adecuada distribución de los mismos. Se han utilizado técnicas de análisis espaciales y de correlación de variables, mediante Sistemas de Información Geográfica, para comprobar si la distribución a los servicios educativos es la adecuada. 
Palabras clave: Educación Infantil; Educación Primaria; Educación Secundaria; localización espacial; secciones censales.

\begin{abstract}
Attending to demographic and socioeconomic characteristics of Extremadura and the Education is one of the most essential universal rights, so it is necessary to ensure its accessibility to the entire population and especially to the most disadvantaged areas. For this reason, in this work we will study the educational resources at an early age (Infant Education, Primary and Secondary Education) in an eminently rural region with low population density, such as Extremadura, to verify if there is an adequate distribution of the same. This work has been done with techniques of spatial analysis and correlation of variables, through Geographic Information Systems, in order to verify if the distribution to the educational services is adequate.
\end{abstract}

Key words: Infant Education; Primary Education; Secondary Education; Spatial Location, Extremadura.

\title{
1 Introducción
}

Las decisiones relativas a la localización de equipamientos o servicios colectivos a la población deberían de ajustarse a los principios de equidad, eficiencia y justicia espacial (Gutiérrez et al., 2000, p. 451; Pitarch, 2000, pp. 130-131) orientando sus decisiones en criterios económicos, sociales y ambientales (Flores \& Rojas, 2009, p. 300) con el objetivo de que la equidad sea conseguir la igualdad de recursos proporcionales al territorio o a la población; la justicia esté ajustada a la necesidad de la demanda de cada espacio y, de igual modo; se obtenga la eficiencia de una adecuada distribución espacial de los servicios para la población. Así pues, una eficiente distribución de recursos permitirá optimizar los desplazamientos totales entre los lugares con demanda y los centros de oferta y su nivel de accesibilidad espacial (Harvey, 1992, pp. 97-99) evitando que el poblamiento sea un factor de diferenciación para el acceso a los servicios que cubren las necesidades vitales de los individuos (Villanueba, 2010, pp. 137-138)

Desde una concepción geográfica se han venido desarrollando o aplicando modelos de localización óptima e índices de accesibilidad que, desde el surgimiento de los SIG, permiten analizar las condiciones actuales y futuras (Fuenzalida \& Moreno, 2010, pp. 453-454) para obtener una disposición adecuada de los equipamientos y servicios y que revierte inevitablemente en el desarrollo regional. Podemos diferenciar tres aproximaciones en los estudios de los equipamientos que pueden condicionar la resolución, selección y análisis: servicios genéricos (sanitario, educativo o cultural) de interés en ámbitos de carácter, nacional o de grandes conjuntos regionales; como objeto (hospital general, hospital psiquiátrico o centro de salud) que responden a 
la orientación del servicio y donde la escala territorial es nacional o regional; y por último, los específicos que aparecen vinculados a la realidad cotidiana de la población, de tal forma que sus ámbitos de estudio pueden ir del regional al comarcal-metropolitano o local-urbano (Calvo et al., 2001, pp. 251-252). La aproximación de nuestra investigación será el estudio del acceso al sistema educativo en etapas tempranas como elemento genérico y en concreto, el análisis como objeto de los diferentes Centros Educativos (Educación Infantil de Primer Ciclo, Educación Infantil de Segundo Ciclo, Educación Primaria y Educación Secundaria Obligatoria) que existen en una sección censal.

La definición de criterios previos y la elaboración de un exhaustivo proceso metodológico constituyen herramientas imprescindibles en la ordenación de cualquier servicio público, con el objetivo de optimizar el uso de los recursos existentes y futuros (Escribano, 2005, pp. 76-77). Dicho procedimiento consta de un análisis, a partir de indicadores cuantitativos sintéticos anteriormente descritos como la eficiencia o equidad espacial; la elaboración de cartografía específica para la visualización de la organización espacial y, finalmente, la determinación de una valoración territorial (Moreno, 2003, pp. 219-222), que en este trabajo se centrarán en los recursos educativos.

El valor de las Tecnologías de la Información Geográfica, y más concretamente los SIG, es incuestionable en la resolución de problemas de ordenación territorial y planificación de cualquier actividad humana (Palomar \& Bosque, 2004, p. 495). Estas herramientas nos permitirán mejorar el conocimiento de la realidad al espacializar, organizar y normalizar la información para una mayor efectividad en la toma de decisiones (Pueyo, 1991, p. 175) y establecer numerosas posibilidades analíticas en el marco de la planificación y gestión de equipamientos o servicios públicos como son: soporte en la gestión operativa de los servicios de planificación de equipamientos, análisis de áreas de servicios; análisis de localización y accesibilidad espacial, etc. (Rodríguez, 2010, p. 561).

Pero nos encontramos con una problemática territorial, no se puede abordar el diseño y la ordenación de un determinado recurso sin tener en cuenta las numerosas divergencias que nos vamos a encontrar en los espacios geográficos; solamente el distinguir entre ámbito urbano y rural puede suponer grandes implicaciones en su organización espacial. La mayoría de estudios realizados para estimar los niveles adecuados en la dotación de equipamientos colectivos, muestran desequilibrios entre los espacios rurales y urbanos (Oberti, 2007, p. 223), ciertamente, las áreas rurales están sujetas a condiciones desfavorables que las relegan como espacios periféricos en relación con el medio urbano, que asume la centralidad como atributo de poder. Los desequilibrios territoriales entre la periferia y la centralidad originan una estructura de regiones favorecidas y desfavorecidas (Reynaud, 1982, p. 425); las primeras, producen una dinámica económica intensa, mientras que las segundas presentan fuertes desigualdades socioeconómicas y demográficas (Izquierdo, 2002, p. 55). Por ello, en este trabajo se estudiará la organización de los recursos en 
una región Extremadura, de carácter predominantemente rural aunque también se localicen centros urbanos y donde esta dualidad rural-urbana en su espacio será tenida en cuenta a la hora de analizar los resultados obtenidos. Existen unas diferencias territoriales, con áreas más dinámicas que se localizan en las zonas agrarias más productivas, en las cuencas sedimentarias explotadas mayoritariamente bien con regadío, bien con secano de vid y olivo, además de ser las zonas mejor comunicadas de la región, y en el lado opuesto, las áreas con menor densidad de población que se localizan en una franja periférica, aislada y menos accesible, con densidades de población que no ocupan, en algunos casos, los $10 \mathrm{hab} / \mathrm{km}^{2}$ y en las que su población no ve satisfechas sus necesidades respecto a calidad de vida y bienestar social y se traslada a aquéllas áreas urbanas o cabeceras comarcales más dotadas, con el fin de buscar mejores oportunidades socioeconómicas y ver cubierta su demanda de equipamientos básicos, como es el caso de los educativos (Nieto \& Cárdenas, 2015a).

En este sentido, también se debe profundizar en el conocimiento del sistema de transportes como un factor primordial en la organización espacial y socioeconómica de los territorios, al mostrar cuestiones que afectan a la cohesión social como las diferencias en la accesibilidad a los servicios y equipamientos colectivos (Linares, 2008, p. 2). La definición de modelos pretende conseguir que la accesibilidad espacial de la demanda (población) a los puntos de servicio sea lo menos dispar posible y para ello se tiende a igualar la oferta desde el punto de vista social y territorial (Gutiérrez et al., 2002, pp. 269-271). Así pues, la inaccesibilidad ocasiona una penalización para aquellos que la sufren, por lo que reducirla al mínimo es prioritario para muchos tipos de equipamientos o servicios (Moreno, 2004, pp. 73-122) como es el caso que nos ocupa, los recursos educativos y para el desarrollo de áreas más desfavorecidas, como son los espacios más ruralizados de Extremadura. El sistema de infraestructuras de la región extremeña, se basa prácticamente en la Red de Carreteras, la cual ha experimentado durante el periodo democrático (1986-2016) los mayores avances tanto cualitativos como cuantitativos, sumiendo al transporte por ferrocarril en un importante retroceso y al aéreo a poseer una presencia meramente testimonial. Dos son las principales arterias que articulan la Red de Carreteras de Extremadura: la Autovía de la Plata o A-66, que discurre de norte a sur y ha actuado como elemento determinante del desarrollo regional al comunicarnos con el norte y el sur de España y, la Autovía A-5, que atraviesa la región de noreste a oeste, comunicando Madrid con Lisboa y en sus zonas más próximas es donde se localizan las áreas más desarrolladas. En el extremo opuesto, existen zonas del norte y este de la provincia de Cáceres y del este y sur de Badajoz muy alejadas por las deficientes comunicaciones, por lo que se ven abocadas a una mayor marginalidad en cuanto a inversiones productivas, localización de equipamientos y servicios y, consecuentemente, concentración poblacional. Por ello, la existencia de una buena accesibilidad es esencial para una buena distribución de los equipamientos y las infraestructuras educativas, de forma que la planificación de la red de Centros de Extremadura se 
debe llevar a cabo de manera que preste un servicio de calidad, atendiendo a las necesidades de la población.

La temática elegida responde a la necesidad de tener un conocimiento fehaciente sobre la accesibilidad que los ciudadanos, tanto en las zonas urbanas como rurales, tienen a los servicios básicos que determinan la calidad de vida, el bienestar social y el proceso de atracción y mantenimiento de la población que presentan sobre todo en los espacios más ruralizados (Escribano, 2011, pp. 21-46). Es una temática de máxima actualidad desarrollado en numerosos trabajos como los realizados en el sector sanitario por Gutiérrez et al (2002), Escalona y Díez (2003), Fuenzalida y Moreno (2010) o Rodríguez (2011) y en el educativo, por Pitarch (2000), Talen (2001), Oberti (2007), Sabuda (2007) o De la Fuente et al. (2013).

Este análisis se circunscribe a las enseñanzas escolares, pues es particularmente en los niveles obligatorios, en las etapas de Educación Infantil, Primaria y Secundaria de Extremadura, donde se presenta de manera más destacada la problemática de ordenar en el territorio una red de Centros, que ofrezca a los ciudadanos un servicio de calidad de forma eficiente y equitativa. Así pues, el objetivo general que se persigue es determinar si la oferta de equipamientos o servicios educativos en la región extremeña es la adecuada para satisfacer la demanda y de ahí radica el interés de este trabajo, demostrar que la planificación educativa puede ser eficazmente abordada desde una perspectiva geográfica. Sobre todo debido a que desde el traspaso de competencias educativas en el año 2000 a la Administración Autonómica se produjo un gran incremento de las inversiones en educación fundamentalmente por la ampliación de la edad de escolarización obligatoria desde los 14 años hasta los 16 años y por la creación de una Red de Centros con construcción de nuevas instalaciones, los Institutos de Enseñanza Secundaria (I.E.S.), sobre todo en áreas rurales menos accesibles para asegurar la Enseñanza Secundaria Obligatoria y la igualdad de oportunidades en todos los contextos (la red de colegios estaba implantada previamente por el Ministerio, centros educativos con población hasta los 12 años). Se pretendió establecer una red moderna y tecnificada de recursos educativos ampliando el número de profesores para mejorar la ratio profesor-alumno, así como por el incremento de las inversiones relacionadas con la sociedad de la información, telecomunicaciones y enseñanza de idiomas. Reseñar también el papel que en el territorio extremeño presenta la escuela rural; la dispersión de su población exige que las zonas rurales sean objeto de especial atención en la planificación educativa con la adecuación de la tipología de los centros y de recursos a sus características, con el fin de recibir una educación en igualdad de oportunidades y en similares condiciones de calidad al resto del alumnado. Por eso, se crearon los "Colegios Rurales Agrupados" configurándose la agrupación de varias unidades de localidades limítrofes en una sola sede denominada cabecera del mismo y, permitiendo equiparar su presencia a niveles "similares" a los de muchas áreas urbanas. 
Reseñar que se estimarán en este análisis como Centros Educativos tanto los Centros Públicos como los Centros Concertados debido a que su acceso en Extremadura es libre y gratuito, y sus criterios de adjudicación de plazas se rigen por las mismos reglamentos que la asignación de los públicos (se prioriza la cercanía, renta y familiares en el centro escolar). No se han estimado centros privados debido a su carácter meramente presencial en esta región (se tendría que añadir 13 Centros Privados que no tienen concertada la educación y son de matrícula de pago).

Por ello, el interés de este trabajo radica en describir estadística y geográficamente la distribución espacial de estos Centros Educativos para localizar inequidades espaciales. Técnicamente se han utilizado a través de Sistemas de Información Geográfica (SIG) sus herramientas de correlación espacial y accesibilidad porque se pretende detectar las agrupaciones espaciales de estos equipamientos (mediante el Índice Local de Moran, el Análisis de Punto Caliente y Densidad Kernel) y si estos óptimos espacios están bien distribuidos en el territorio extremeño, a través del cálculo de tiempos mínimos de acceso a los mismos. Los resultados muestran un diagnóstico espacial de la calidad educativa en el área de estudio, así como las zonas de menor y mayor concentración de equipamientos. Se obtendrá la detección de puntos débiles en la oferta de servicios, cambios demográficos y territoriales acontecidos, para posteriormente se puedan aplicar las medidas oportunas para resolver estas deficiencias detectadas (Prat et al., 2008, p. 13).

\section{Metodología}

Conforme a nuestro objetivo general de reflexionar sobre la distribución de los equipamientos educativos en etapas de Educación Obligatoria en Extremadura, se ha desarrollado este trabajo en las siguientes etapas: almacenamiento de la información y generación de la base de datos; análisis estadístico para estimar la relación entre variables de oferta y demanda; estudio de accesibilidad; identificación de patrones geográficos y presentación de resultados y conclusiones donde se detectan las fortalezas y debilidades del sistema educativo.

\subsection{Generación de la Base de Datos de Recursos Educativos}

En este hilo conductor, los elementos determinantes en el análisis y ordenación de los equipamientos educativos son: la demanda, definida como los individuos que desean utilizar el servicio público y que se asientan en un lugar concreto, y la oferta, condicionada por las zonas en las que se sitúan las instalaciones educativas. Por ello, para determinar la oferta, si los servicios llegan a atender a todas las personas que son potencialmente usuarios, se han utilizado los datos proporcionados por la Consejería de Educación de la Junta de Extremadura para el curso 20142015. La información ha sido facilitada en una base de datos con una relación del conjunto de Centros de Extremadura, su número de matrículas y unidades (espacios-aulas con la correspondencia de al menos un profesor por cada 25 alumnos en Educación Primaria y 30 
alumnos en Educación Secundaria Obligatoria). Para este estudio se han seleccionado las etapas de Educación Obligatoria e Infantil menor de 3 años por considerarse servicios básicos que determinan la calidad de vida y el bienestar de la población. Los ciclos de enseñanza valorados se estructuran de la siguiente forma: Educación Infantil de Primer ciclo (0-2 años); Educación Primaria (5-11 años) y Educación Secundaria Obligatoria (12-16 años). Los datos referidos a Educación Infantil de Segundo ciclo (3-5 años) no se han introducido como variable independiente ya que se ha comprobado previamente que el $95 \%$ de los Centros Educativos de Extremadura que ofertan Primaria también ofertan la Infantil de Segundo Ciclo al haberse considerado como una oferta complementaria a la misma desde la obtención de las transferencias educativas regionales en el año 2000. Por su parte, los Centros de Educación Infantil de Primer Ciclo si se han analizado como variable independiente y se corresponden con las guarderías de carácter público.

El siguiente paso fue realizar un proceso de georreferenciación para una localización puntual de los equipamientos educativos de Extremadura de las etapas seleccionadas obteniéndose las coordenadas con la herramienta de geo-visualización Google Earth para, a continuación, comprobar los resultados con el visor del Instituto Geográfico Nacional, Iberpix.

Para estimar la demanda de este recurso, se elaboró un segundo estrato de información, la población demandante de este servicio calculándose por grupos de edad de cada etapa educativa la población a nivel de sección censal de Extremadura. Se obtuvo del Padrón del 2015 y se añadió a la base cartográfica con estructura topológica de SIG de las secciones censales, ambos datos obtenidos del Instituto Nacional de Estadística. Se decidió realizar el análisis por secciones censales debido a las peculiaridades del territorio de Extremadura, con municipios de gran extensión superficial donde coinciden el núcleo principal, las entidades menores de población y las pedanías (Nieto \& Cárdenas, 2015b, p. 223). La cartografía digital a fecha de 2015 cuenta con un total de 970 secciones censales para la región de Extremadura y a través del Código de Sección Censal establecido por el INE (un código numérico de 10 dígitos) se le añadieron los indicadores demográficos para posteriormente elaborar el dato estadístico que se va a emplear como indicador de la demanda de recursos educativos: el Índice de Juventud (estos datos quedan asociados a la tabla de atributos con la división en polígonos de las secciones censales de Extremadura).

El proceso siguiente fue asignar a las secciones censales que tenían ya añadidos los indicadores demográficos (demanda) las instalaciones educativas localizadas en cada una de ellas (oferta), agrupándose el número total de Centros; unidades educativas y matrículas por cursos académicos mediante la suma absoluta de dichas variables para cada elemento poligonal.

Posteriormente para poder realizar análisis de accesibilidad o localización espacial esta capa poligonal de secciones censales se transformó a una capa de puntos. Se trata de la asignación a cada uno de los polígonos de las secciones censales de un punto representativo que, en este caso, 
se corresponde con un punto central. Uno de los problemas de su utilización deriva del propio criterio de localización del punto, que ha sido necesario situar en relación al núcleo urbano principal y no según la superficie total de la sección censal. Esta capa de puntos, con los centroides de las 970 secciones censales, representa la demanda potencial o la capa de los orígenes. Se decidió establecer la capa de secciones como puntos debido a la distribución espacial de Extremadura, grandes extensiones de territorio en una sección censal o municipio donde la población sólo se localiza en el núcleo principal, en este caso en el territorio de la sección censal localizado en el mismo. De ahí, por ejemplo, la baja densidad demográfica en los espacios urbanos como Badajoz - Cáceres que son las entidades municipales más extensas de España y que con este indicador deberían establecerse como zonas rurales cuando se concentran sólo en el núcleo principal los 150000 o 95000 habitantes en cada caso.

La última capa cartográfica que se obtuvo fueron las vías de comunicación, en este caso las carreteras, obtenidas del Mapa Oficial de Carreteras del año 2013 del Ministerio de Fomento. Se decidió no incluir otros medios de desplazamiento públicos, como el ferrocarril y el aéreo, al no emplearse en el traslado diario de escolares en el territorio extremeño.

Para estimar la relación entre la demanda y la oferta el primer análisis que se estableció fue el coeficiente de correlación de Pearson (1896) como una medida de asociación lineal adecuada para estudiar la relación entre variables de intervalo o razón (cuantitativas). Está representado por r y es resultado de tipificar el promedio de los productos de las puntuaciones diferenciales de cada caso (desviaciones de la media) en las dos variables correlacionadas (González, 2007, pp. 140-145), en este caso la población menor de 16 años y la suma de unidades ofertadas en cada sección censal. Se obtendrán valores comprendidos entre -1 y 1: un valor de 1 señala una relación lineal perfecta positiva; un valor de -1 indica relación lineal perfecta negativa (en ambos casos los puntos se encuentran dispuesto en línea recta); un valor de 0 muestra una relación lineal nula. El coeficiente $r$ es una medida simétrica, es decir, la correlación entre Xi e Yi es la misma que entre Yi y Xi. Asimismo, el signo del coeficiente informa sobre la dirección de la relación y su valor absoluto marca la fuerza (Martínez, 2013, p. 286).

Una vez establecido si existe relación entre la demanda y oferta de Centros nuestro objetivo será aplicar diversos métodos de análisis espacial para examinar la distribución de los mismos, siendo la accesibilidad geográfica, la densidad de áreas Kernel y la localización de agrupamientos o clusters con el I Anselin Local de Moran y Getis-Ord. Se decidió fundamentarnos en cuatro indicadores: el número de Unidades ofertadas por sección censal en todos los Centros Educativos, en $1^{\circ}$ de Educación Infantil, $1^{\circ}$ de Educación Primaria y $1^{\circ}$ de Educación Secundaria. Se establecieron éstos por ser los definitorios de cada etapa educativa, si se conceden dos unidades en $1^{\circ}$ de Educación 
Primaria ser irán manteniendo en los siguientes cursos de esta etapa educativa, hasta sexto inclusive, y con la Educación Secundaria y la Infantil de $1^{\circ}$ Ciclo se produce la misma casuística.

\subsection{Análisis de Accesibilidad}

Para acometer el análisis de accesibilidad geográfica desde la población que vive en las distintas secciones censales extremeñas a los recursos educativos, se ha utilizado una metodología basada en un estudio de redes empleando las funciones de análisis que presentan los SIG. Así, el análisis de redes se encuadra en los postulados de la teoría de localización, pues examina la localización actual y la distribución de los centros de enseñanza en relación con el asentamiento de la población. Bajo esta realidad, debemos detectar las áreas con distintos grados de accesibilidad a los servicios para determinar la población en riesgo de exclusión (Ramírez, 2007, p. 1).

Una red es un sistema de arcos interconectados por la que pueden ponerse en movimiento flujos de distinto tipo. Los elementos de una red son los nodos, en nuestro estudio los centros de educación y los respectivos núcleos de población (centroides de las secciones censales), conectados por arcos o aristas, en este caso las carreteras. El modelo de análisis de redes se apoya en la teoría de grafos que responde a un esquema lineal que sirve para precisar la estructura topológica de la red, con una serie de características: longitud, dirección, conectividad, impedancia o restricciones de acceso. Para el cálculo de estos índices es necesario conocer la impedancia, es decir, el efecto de resistencia que se asocia al desplazamiento por la red y que se ha calculado teniendo en cuenta la longitud (en Km) y la velocidad de las vías (la máxima permitida atendiendo a si son carreteras locales, nacionales o autovías) en la siguiente expresión:

$$
\text { [Longitud]/([Velocidad]*1000)/60) }
$$

Se trata de calcular el tiempo de desplazamiento mínimo desde un origen (población de las secciones) al destino más cercano (centros de enseñanza) porque el tiempo de recorrido se ha convertido en un elemento de mayor relevancia para el estudio de movilidad (Rodríguez, 2011, p. 270). En síntesis, el planteamiento metodológico consiste en entender la potencial interacción entre la población (demanda) y las instalaciones educativas (oferta) presentes en Extremadura con el objetivo de valorar la equidad en la distribución de los servicios básicos. El cálculo del tiempo de acceso mínimo de cada centroide al recurso educativo más próximo mostrará un modelo ideal en el que la población siempre se acerca al núcleo más cercano sin tener en cuenta las funciones que desempeña ni la competencia con otros aunque más alejados. La expresión es la siguiente:

$$
\mathrm{TAMi}=\min \left(\mathrm{IR}_{\mathrm{ij}}\right) \forall \mathrm{j}
$$

donde se averigua el tiempo mínimo de cada nodo i (centroide de cada sección censal) a la instalación educativa más cercana (nodo j). La cobertura lineal muestra los tramos de carreteras de la red viaria incluyendo, como información alfanumérica, la impedancia en minutos siendo el tiempo 
mínimo de acceso desde los centroides de las 970 secciones censales a los equipamientos educativos más cercanos obtenidas con la herramienta "Closest Facility" del software ArcGis. Posteriormente, estos datos han sido asignados a la capa de puntos de las secciones censales para aplicar el método de interpolación IDW, y obtener una superficie raster utilizando la distancia inversa ponderada ("Inverse Distance Weight"). Es una técnica de interpolación determinística que fija valores a las localizaciones, basándose en las medias de los valores circundantes. Con este método de interpolación la variable de tiempo mínimo, representada cartográficamente en una imagen ráster con distintos valores, disminuye su influencia a mayor distancia desde su localización y a la inversa.

\subsection{Análisis de Localización Espacial}

Algunas de las técnicas estadísticas más utilizadas para el análisis espacial y la geoestadística son el indicador de autocorrelación parcial I Anselin Local de Moran y el análisis de punto caliente Gi* de Getis-Ord como parte del conjunto de métodos cuantitativos que tienen por objeto la identificación y la descripción de patrones de distribución geográfica. Son herramientas eficaces para el análisis de patrones, la medición de distribuciones geográficas, el modelado de relaciones espaciales o la asignación de clústeres para agrupar objetos en base a las características de los mismos (Hair, 2008, p. 492). Para abordar esta temática de investigación se ha considerado necesario aplicar los indicadores citados a las cuatro variables determinadas anteriormente y obtener información sobre su patrón general utilizando las herramientas de estadística espacial disponibles en el Sistema de Información Geográfica ArcGis 10.2.

Un concepto esencial en estas técnicas de análisis espacial es el de dependencia o autocorrelación, que aparece como resultado de la existencia de un vínculo entre lo que sucede en un lugar determinado del espacio y lo que ocurre en otro lugar (Rivera, 2014, p. 19) para delimitar el grado en que objetos o actividades en una unidad geográfica son similares a otros como consecuencia de su proximidad (Vilalta \& Perdomo, 2005, p. 325). Este supuesto conecta la estadística espacial con los trabajos de Tobler (1979) y su "primera ley de la Geografía" en la que se sostiene la idea de que todo está relacionado con todo en el análisis geográfico, pero las cosas cercanas están más relacionadas entre sí que las cosas lejanas (Carles \& Hoberg, 2008, p. 2). Por tanto, existe una autocorrelación espacial positiva cuando los objetos cercanos se parecen mucho entre sí; en el supuesto contrario, hablamos de una autocorrelación espacial negativa cuando los objetos difieren mucho unos de otros por el hecho de estar juntos. La correlación será nula en el hipotético caso de que no existe relación entre la aparición de un hecho y el lugar que ocupa.

Estos procedimientos experimentaron un gran avance con el desarrollo del I Anselin Local de Moran (LISA, Local Indicators of Spatial Associacion) en un software SIG; que descompone el Índice global de Moran, verificando en cuánto contribuye cada unidad espacial a la formación del 
valor general y capturando el grado de asociación espacial y la heterogeneidad resultante del aporte de cada unidad espacial (Celemín, 2009, p. 28). Así esta técnica facilita la exploración visual de agrupamientos o patrones territoriales formados en función de los datos de una variable que muestran las unidades de observación analizadas y las unidades vecinas (Iris, 2015, p. 144).

Se obtiene un valor I de Moran Local para cada registro introducido (en este estudio las unidades de cada etapa educativa) con una puntuación $z$, un valor $\mathrm{P}$ y un código que representa la entidad estadísticamente significativa. Estas puntuaciones se representarán en un código que nos indica: zonas con presencia alta de miembros de un grupo rodeadas de zonas con presencia también alta (situación High-High), o bien zonas con presencia alta rodeadas de unidades con presencia baja (situación High-Low). Asimismo, también se permite la detección de zonas con presencia baja rodeadas de unidades también con presencia baja (situación Low-Low), o bien zonas de presencia baja rodeadas de unidades con presencia alta de la variable analizada (situación Low-High). Por último, también se pueden detectar zonas sin asociación espacial significativa (Anselin, 1995). Sin duda, obtener un número que pueda cuantificar el patrón espacial clúster y dónde se pueden producir agrupaciones en la distribución de las unidades educativas es prioritario en este estudio.

Por otro lado, el análisis de Getis-Ord indica si las funciones con valores altos o valores bajos tienden a concentrarse en un área de estudio identificando clusters espaciales estadísticamente significativos de valores altos (puntos calientes) y valores bajos (puntos fríos) respecto al promedio de la muestra. Evalúa cada entidad dentro del contexto de entidades vecinas y comparan la situación local con la situación global (promedio de unidades educativas). El resultado es una puntuación Z, para las puntuaciones positivas estadísticamente significativas, medidas de desviación estándar, por ejemplo, si una herramienta devuelve una puntuación $Z$ de 2,5 se interpreta como "2,5 desviaciones estándar de la media" (Getis and Ord, 1992). En efecto, para ser considerado como un punto caliente estadísticamente significativo, una entidad debe de cumplir dos condiciones: tener un valor alto y estar rodeada por otras entidades con valores altos respecto al promedio y la localización espacial de entidades con estas características refleja dónde se producen los aglomerados y el grado de confianza estadística (Galacho y Reyes, 2015: 607608).

Para completar estos análisis se ha utilizado el cálculo de la Densidad Kernel en las mismas variables, un estadístico que, a partir de los puntos de muestreo o entidades, calcula las magnitudes de las áreas donde se pueden producir los aglomerados. Es decir, los dos métodos anteriores nos localizarán la presencia alta o baja de puntos con localización de unidades educativas, pero con este método se obtendrán áreas donde se localizan agrupaciones de estos puntos. El método Kernel es una forma de evaluar la intensidad; si el patrón espacial de distribución tiende a presentarse en agregados, mostrará distintas densidades en diferentes zonas, pues este tipo de análisis espacial calcula la densidad de puntos por unidad de área. De este modo, el 
estimador Kernel conforma una superficie curvada suave sobre cada punto utilizando cálculos de vecindad local fijada por bloques de celdas o pixeles y una función simétrica radial (De Cos, 2004: 144). El valor de densidad es más alto en la ubicación del punto y tiende a disminuir progresivamente a medida que aumenta la distancia desde el punto, hasta el radio máximo de búsqueda (bandwidth) donde el valor de densidad llega a cero.

\section{Resultados y discusión}

La aplicación de la metodología propuesta para los datos disponibles nos ha permitido extraer conclusiones de gran calado que sucintamente se procede a desglosar y valorar en este epígrafe.

\section{Figura 1. Índice de Juventud y Localización de Centros Educativos}

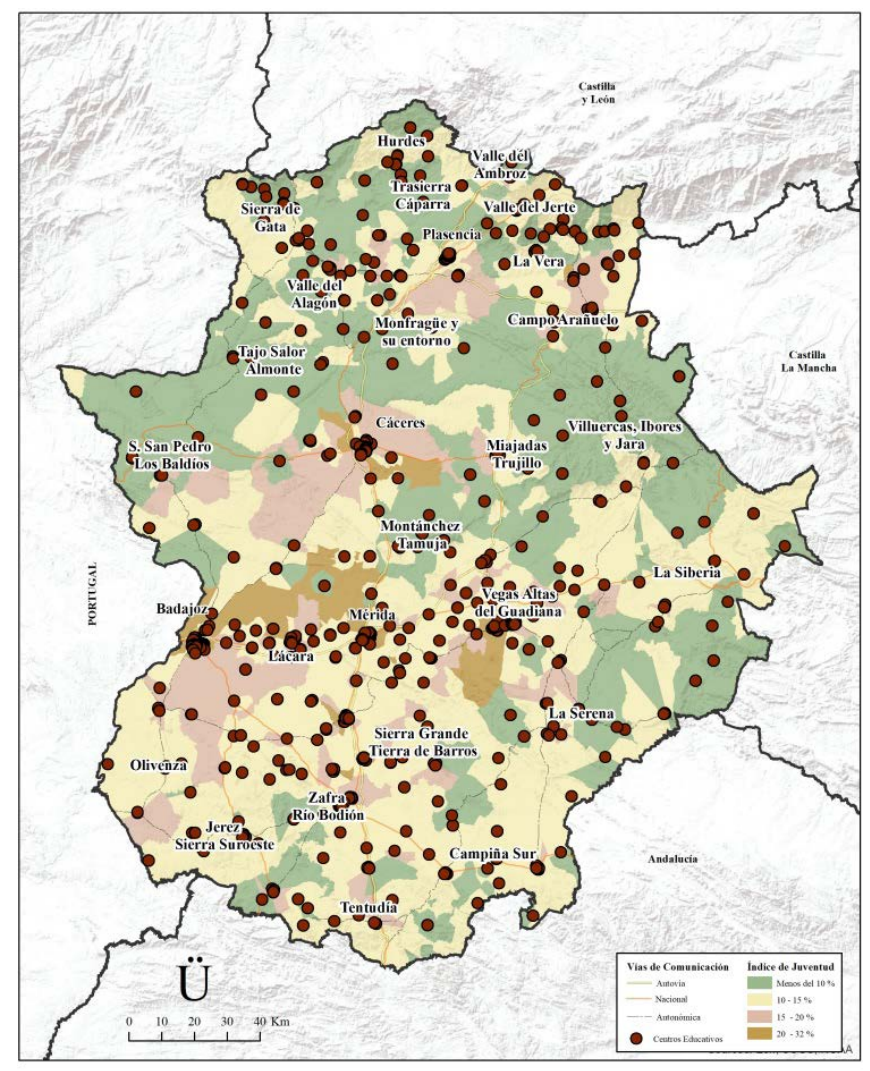

Fuente: elaboración propia a partir de los INE y de la Junta de Extremadura (2015)

El análisis de indicadores demográficos de juventud en Extremadura permite una comprobación del incremento en los contrastes internos de este espacio y la progresiva consolidación de un modelo de distribución poblacional caracterizado por la concentración en áreas puntuales y cada vez más limitadas del territorio regional, los valores más elevados. En la Figura 1, donde se muestra el Índice de Juventud de las secciones censales, los valores más elevados quedan concentrados fundamentalmente en las siguientes áreas: los núcleos urbanos de Cáceres y Badajoz, las zonas de regadío del río Guadiana en la provincia de Badajoz y del Tiétar en la provincia de Cáceres (con un valor superior al $16 \%$ ). Asimismo, existen secciones censales con valores altos dispersos en 
otros territorios agrarios intensivos como el regadío y el secano productivo de vid y olivo localizado en áreas de Olivenza, Tentudía, Zafra-Río Bodión, Barros Oeste-Sierra Grande y Valle del Alagón (con una concentración de secciones censales que arrojan datos de juventud entre 11-15\%). Sin embargo, es sobre todo en la provincia de Cáceres donde se aglutinan aquellas divisiones que presentan datos inferiores al $10 \%$ en la variable analizada, en zonas pertenecientes a las comarcas de la penillanura trujillano-cacereña y las áreas de montaña del norte de Cáceres (Sierra de Gata, Hurdes, Trasierra-Cáparra, Ambroz; Valle del Jerte y La Vera), Villuercas y Sierra de San Pedro (Figura 1). Es un elemento incuestionable que la oferta de equipamientos y servicios tienden a disponerse en los asentamientos urbanos y en determinados núcleos rurales pero se debe determinar si existe una relación estadística significativa entre la localización de estas Unidades Escolares y la demanda potencial de población joven (menores de 16 años). Por ello, se aplicó el coeficiente de correlación de Pearson para conocer el nivel de dependencia entre ambas entidades. Se parte de la premisa que pueda existir una relación lineal entre las dos entidades, de modo que los valores del sujeto "Suma de unidades" dependerá de la puntuación del indicador demográfico "población menor de 16 años", como variable independiente, y por ello se ha calculado un diagrama de dispersión para la representación de las dos variables cuantitativas, de modo que podamos definir el tipo de relación lineal existente.

Este gráfico (Figura 2) ilustra de forma clara la inexistencia de una pauta de variación evidente, lo cual se refleja por la dispersión de la nube de puntos, muy lejos de asemejarse a una línea recta. Otro aspecto a destacar es el valor que toma el R2 Lineal que es de 0,0745 un dato bastante bajo y poco explicativo teniendo en cuenta que tenemos muchas casillas que provocan distorsiones al sistema, como las secciones censales que presentan cero unidades escolares. Es decir, nuestro modelo tiene una explicación del 4,7\% que expresa cuánto del valor de la variable dependiente "Suma de unidades" está determinado por la variable independiente "Población menor de 16 años".

Por otro lado, el resultado del coeficiente de correlación de Pearson ( $r$ ) entre el total de unidades escolares presentes en cada una de las secciones censales de Extremadura y la población menor de 16 años correspondiente se obtiene una cifra de 0,216. Así pues, debemos interpretar que existe una relación débil entre las variables al encontrarse el valor de 0,216 (r) más próximo de 0 (relación lineal nula) que de 1 (relación lineal perfecta positiva).

En función de los datos obtenidos se puede determinar que la Red de Centros Extremeños no ha sido diseñada en relación al número de jóvenes que demanda este tipo de recurso, sino más bien en función de la accesibilidad geográfica para intentar asegurar que toda la población pueda acceder a la Enseñanza Obligatoria en igualdad de condiciones. Por ello, no son representativos ni 
el Gráfico de Regresión Lineal ni la correlación entre las variables de juventud y el número de unidades ofertadas.

Figura 2. Gráfico de Regresión Lineal

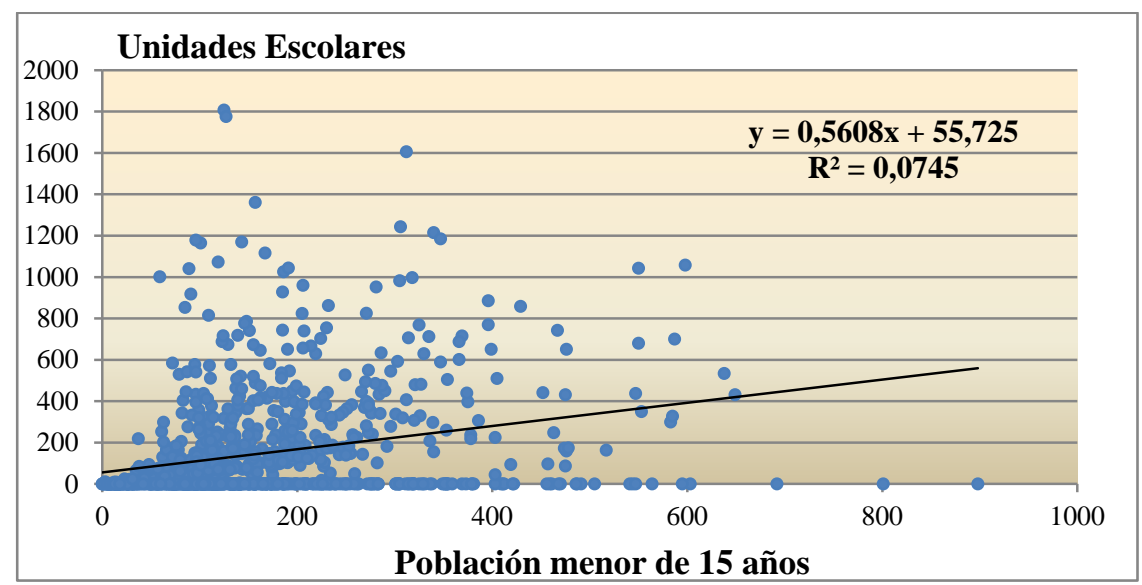

Fuente: elaboración propia a partir de los INE y de la Junta de Extremadura (2015)

Como se ha comentado en epígrafes anteriores, la planificación educativa debe encaminarse, en lo que respecta a la distribución y zonificación de los servicios educativos, a la búsqueda de un modelo de igualdad de acceso. Por ello, los resultados que se obtienen con el análisis de redes nos permiten valorar la accesibilidad geográfica a los Centros Educativos desde cada una de las secciones censales (centroides); es la determinación del coste de acceso a microescala o de micromovilidad (Figura 3).

Una mirada detallada de los datos permite reconocer diferencias de accesibilidad a distintas escalas y algunas divergencias en el acceso respecto a la tipología o variable de estudio; es decir, el tiempo mínimo difiere notablemente si consideramos un centro de Educación Primaria o uno de Educación Secundaria Obligatoria, pues existe un mayor número del primer tipo que del segundo en el territorio extremeño. Con todo, la accesibilidad media a los Centros Educativos (655) de la Comunidad desde cada uno de los centroides de las secciones censales (970) puede considerarse adecuada (2,7 minutos). El $57 \%$ de las secciones se localizan a una distancia igual o inferior a los 2 minutos al servicio educativo más próximo (63 \% de la población de Extremadura); el 19 \% de las entidades están contenidas en la franja de entre 2-4 minutos mientras que el $24 \%$ restante se encuentra en un recorrido superior a los 4 minutos. Si ordenamos las secciones censales de mayor a menor tiempo de acceso, y en consecuencia de mejor a peor accesibilidad, se aprecia que con el valor de tiempo más bajo (0 minutos) están las cabeceras administrativas con vocación de centralidad (secciones censales de núcleos de más de 10000 habitantes), que aglutinan la mayor parte de los equipamientos. Nos referimos a zonas próximas a los principales ejes de comunicación de Extremadura (Autovías a Madrid y Ruta de la Plata), que articulan el territorio de norte a sur y de 
este a oeste. Los valores más altos (superior a los 20 minutos) se localizan en secciones censales que pertenecen a las zonas de montaña o penillanura, en los extremos de la región y más alejadas de las dos principales vías de comunicación (Sierra de San Pedro; Sierra de Gata; Hurdes y Trasierra en la provincia de Cáceres y La Siberia; La Serena y Campiña Sur en la provincia pacense). Del mismo modo, se ha obtenido también una comparación de la variable accesibilidad desde cada una de las secciones censales de Extremadura a los distintos Centros de Educación (Figura 3).

Figura 3. Accesibilidad a los Centros Educativos Extremeños

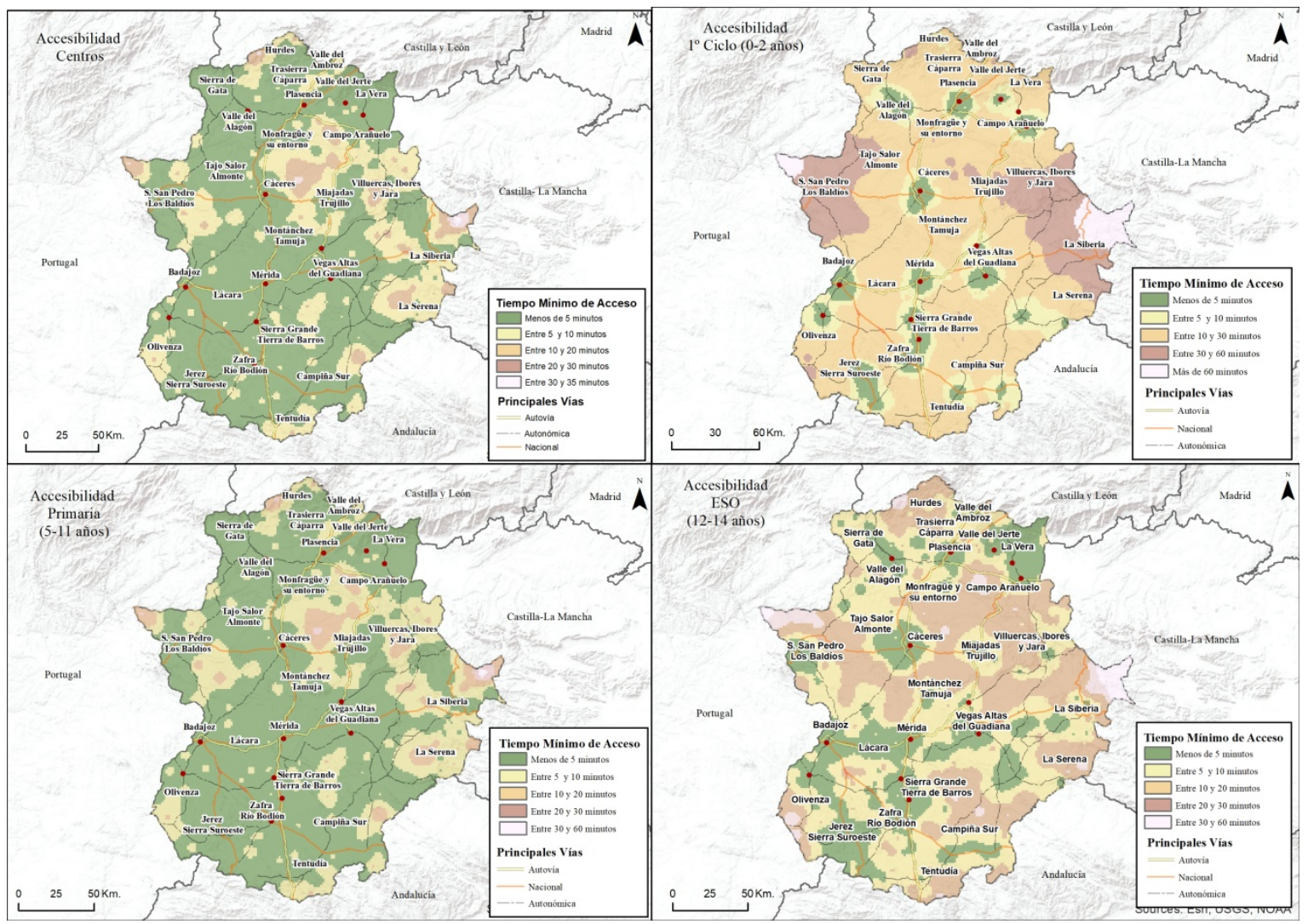

Fuente: elaboración propia a partir de los INE y de la Junta de Extremadura (2015)

Para los Centros de Infantil de Primer Ciclo (0-3 años, 42 centros) su distancia media en tiempo es de 12 minutos, donde el $40 \%$ de las secciones censales se encuentran a una distancia inferior o igual a 5 minutos (48\% de la población); el 24\% entre 5-15 minutos y un 22\% entre 15-25 minutos. Efectivamente, esta tipología de Centros se ubica en las principales ciudades o polos de atracción de la región en relación al sinuoso trazado de las vías de comunicación, como factor determinante. Las secciones censales que se sitúan a más de 25 minutos del Centro de Educación Infantil de Primer Ciclo más cercano suponen el 14\% del total; algunas de ellas superan los 65 minutos y quedan incluidas en los términos municipales de la frontera con Portugal y Castilla LaMancha, más acusada en la provincia cacereña. 
En los Centros de Educación Primaria de Extremadura (466) el tiempo medio de acceso es de 3 minutos (Figura 4); el $56 \%$ de las entidades se encuentran en el primer intervalo, a una distancia inferior o igual a los 2 minutos (el $62 \%$ de la población total), el $19 \%$ entre 2-4 minutos; un $10 \%$ en el rango comprendido entre los 4-6 minutos, mientras que únicamente un 15\% de la secciones censales están a una distancia-tiempo que excede los 6 minutos. Los valores más altos se corresponden con entidades de municipios de la penillanura trujillano-cacereña y de las áreas fronterizas, pero no superándose los 30 minutos.

Por último, en la evaluación de la accesibilidad a los equipamientos del nivel de Educación Secundaria Obligatoria (12-16 años), se evidencian peores datos que para la variable anteriormente analizada (Educación Primaria) pero mejores que para Educación Infantil. Teniendo en consideración que la población afectada, entre 12-16 años, presenta mayores posibilidades de desplazamiento en transporte escolar público por su madurez, por ello, los resultados siguen siendo óptimos en la mayoría del territorio que no superan los 15 minutos. Según el análisis de redes, el tiempo medio de acceso calculado para el conjunto de secciones censales es de 5 minutos; efectivamente, podemos matizar que un 53\% de las entidades (63\% de la población extremeña) se encuentran en un recorrido inferior o igual a los 4 minutos del Centro de Educación Secundaria más próximo (191). En el segundo intervalo se incluyen el $19 \%$ de las secciones con una distanciatiempo de 4-8 minutos; los valores para el 13\% de las secciones son de 8-12 minutos. No obstante, el 15\% de las entidades que presentan un tiempo de acceso superior a los 12 minutos representan el 9,3\% de la población (Figura 2). Los valores más altos se corresponden con secciones censales de áreas de montaña del norte de Cáceres y de la frontera con Portugal y Castilla La-Mancha, pero con valores más elevados que en la educación Infantil de Primer Ciclo (entre 45 y 60 minutos de desplazamiento).

Con este primer análisis se han detectado zonas con algunas deficiencias de acceso a los recursos educativos, más acusados en la variable de Educación Secundaria que en Primaria. Existe un mayor número de centros y unidades escolares de edades tempranas para que estos alumnos no tengan que realizar grandes desplazamientos y se ha fomentado con la aparición de Colegios Rurales Agrupados y la creación de nuevos centros en municipios menores que su población no sea marginada con respecto a los núcleos urbanos o mejor localizados de la región extremeña. Sin duda, se ha priorizado la construcción de Centros de Educación Primaria pues al tratarse de alumnos de entre 5-12 años la población demanda la proximidad de estos centros a su lugar de residencia. Con este análisis se observa la todavía excesiva dependencia del territorio extremeño en accesibilidad a un sistema de transportes desarticulado y fundamentando en los dos ejes que cruzan la región de norte a sur y de este a oeste (las autovías Ruta de la Plata y la A-5 a Madrid). 
Para completar el análisis de accesibilidad, donde se estudiaba la localización del Centro Educativo pero no la oferta de unidades $y$, estimar si existe una mayor oferta de unidades en aquellos territorios de mejor acceso espacial se calcularon análisis de patrones espaciales con las técnicas de I Anselin local de Moran, Gi* de Getis-Ord y de Densidad de Kernel para estos indicadores espaciales seleccionados.

En primer término, se ha calculado la autocorrelación espacial de las unidades escolares en las distintas etapas educativas según la técnica LISA (Anselin) con el objetivo de obtener estadísticas para cada área que nos suministren información sobre la relevancia de valores similares alrededor de la misma. En el total de Unidades Escolares se han identificado zonas con valores elevados rodeadas de entidades también con valores altos (High- High) en 20 secciones censales, ellas pertenecientes a las zonas urbanas más dinámicas de Extremadura: Cáceres, Badajoz, Mérida, Almendralejo y el caso específico de Talayuela (un núcleo que ha recibido gran volumen de población emigrante en las últimas décadas y ha rejuvenecido sus efectivos) (Figura 4).

\section{Figura 4. I Anselin Local de Moran de Centros Educativos Extremeños}

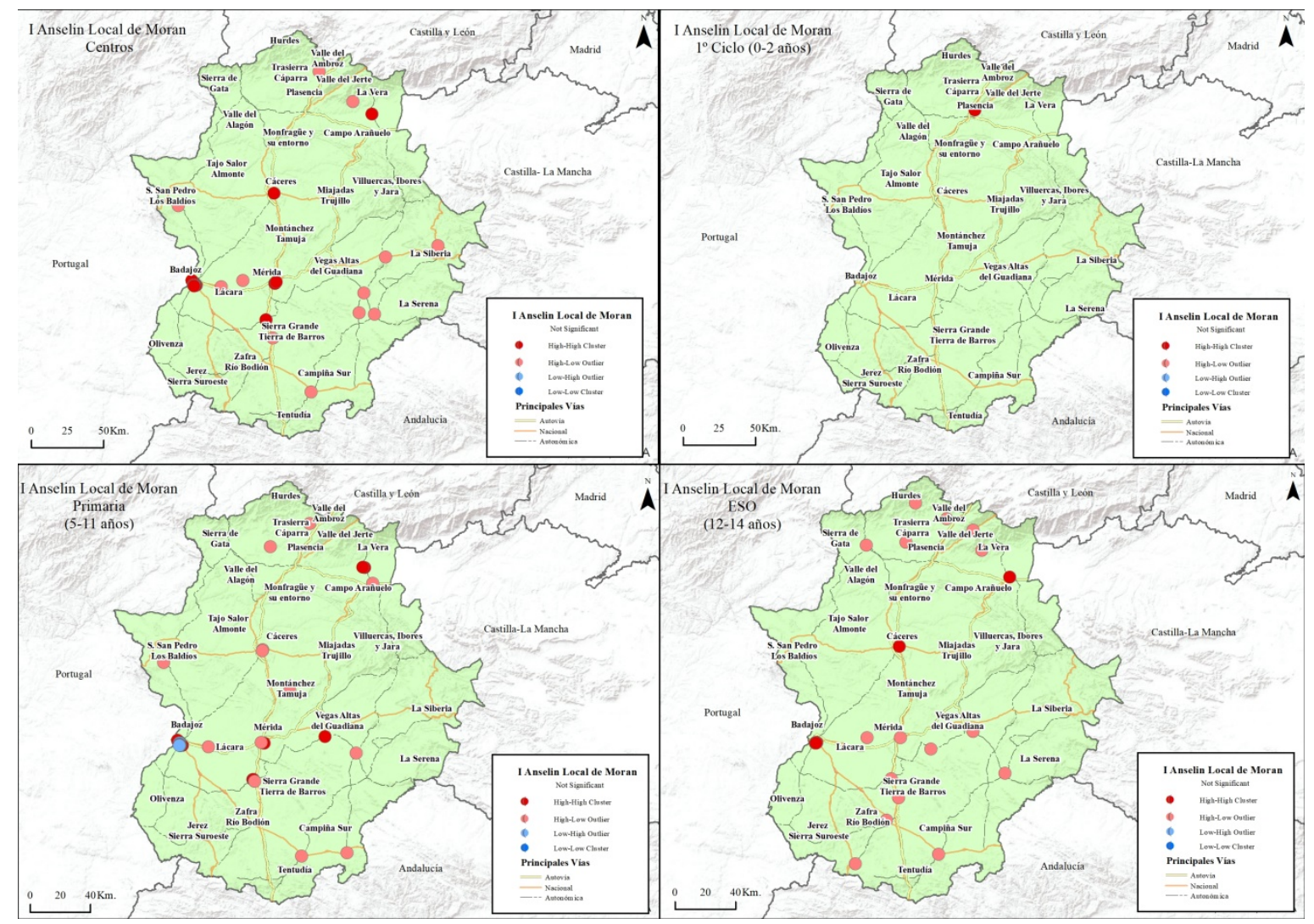

Fuente: elaboración propia a partir de los INE y de la Junta de Extremadura (2015)

La relación alto-bajo o High-Low muestra agrupaciones de secciones censales con valores altos de la variable analizada rodeada de entidades con valores bajos. Estos datos se han registrado en 12 de las 970 secciones censales extremeñas, en las comarcas de Sierra de San Pedro-Los Baldíos 
(San Vicente de Alcántara); Trasierra-Cáparra (Zarza de Granadilla); La Vera (Jaraíz de la Vera); La Siberia (Navalvillar de Pela y Herrera del Duque); La Serena (Campanario, Castuera y Quintana de la Serena); Campiña Sur (Llerena); Barros Oeste- Sierra Grande (Villafranca de los Barros) y Lácara (Talavera la Real y Montijo). Asimismo, y con escasa relevancia en el conjunto muestral, se observan 4 secciones censales con presencia baja de Unidades escolares rodeadas de zonas con valores elevados (Low-High); este cluster de secciones se encuentra en el término municipal de Badajoz. En las restantes 934 secciones censales de Extremadura sometidas a estudio no se observa asociación significativa para la variable "Unidades escolares". Con estos datos, se puede afirmar que existen valores similares de oferta de Unidades Escolares en gran parte del territorio extremeño, a excepción de los supuestos mencionados que se corresponden con las secciones censales de los municipios de más de 10000 habitantes que presentan una concentración de valores altos en determinados espacios.

En el acceso a Educación Primaria, los valores de las agrupaciones son similares destacando además las cabeceras comarcales de algunas áreas más despobladas donde se localizará la oferta de Unidades Escolares para los Colegios Rurales Agrupados, por ello se obtiene una situación High-Low de entidades con presencia alta de miembros rodeadas de secciones con valores bajos.

Para la variable de la Educación Secundaria se obtienen resultados con alta significancia con la relación alto-alto (High-High) donde muestra clústeres de secciones censales (6) con valores altos rodeadas por entidades también con valores elevados: Navalmoral de la Mała, Badajoz y Cáceres capital. De igual modo, la aplicación de la técnica LISA ha determinado la presencia de zonas con una relación alto-bajo (High-Low); nos referimos a entidades de población (16) con un valor elevado en el número de Unidades para Secundaria rodeadas por unidades con escasa presencia de muestras. Se localizan en el área más septentrional de la región (en las cabeceras comarcales de las Hurdes, Valle del Alagón, Gata, Trasierra o La Vera como son Caminomorisco, Moraleja, Montehermoso, Zarza de Granadilla, Navaconcejo y Jaraíz de la Vera) y la zona central de la mitad sur (Mérida, y las cabeceras comarcales de las Vegas del Guadiana, Barros Oeste, Zafra-Río Bodión, Tentudía como son Guareña, Villanueva de la Serena, Castuera, Almendralejo, Villafranca de los Barros, Zafra, Fregenal de la Sierra y Llerena). Los centros de Educación Secundaria Obligatoria son demandados por jóvenes de entre 12-16 años que, desde localidades menores, se desplazan diariamente en autobuses ofertados por el Gobierno de Extremadura a la cabecera de la comarca o núcleos próximos, donde se localizan los institutos de enseñanza (Figura 4).

Los resultados cartográficos obtenidos del procesamiento de la información de la variable del total de las Unidades Escolares con el análisis de Getis-Ord son de indudable importancia. Los puntos calientes o Hot Spot indican las zonas en las que los valores de unidades son altos respecto al promedio de unidades escolares y se concentran en los núcleos o polos centrales de mayor 
prestación de servicios de Extremadura. Las secciones censales con un porcentaje de significación de $99 \%$ se ubican en los municipios de Cáceres, Badajoz, Plasencia, Mérida y en las cabeceras comarcales de Campo Arañuelo, Vegas del Guadiana, Barros Oeste-Sierra Grande, Campiña y La Vera. Por su parte, con un nivel de significación del $95 \%$ localizamos secciones censales también pertenecientes a los términos municipales de los cuatro núcleos urbanos: Cáceres, Badajoz, Plasencia, Mérida, y otras cabeceras comarcales de las áreas anteriores además de incluir Olivenza, Sierra de San Pedro, La Serena y La Siberia. Los puntos calientes con un porcentaje de significación de $90 \%$ se incluyen en localidades menores dispersas por el territorio extremeño. No se encuentran agrupaciones de valores en secciones censales con datos de puntos fríos porque no se localizan áreas extensas donde no exista oferta escolar significativa. Esto nos muestra que la oferta de unidades escolares está diseminada por el territorio aunque se localicen determinadas concentraciones en las áreas más urbanas, dinámicas y con mayor población joven de la región (Figura 5) donde el análisis de punto caliente con Getis-Ord ha identificado 78 puntos calientes (Hot Spot) repartidos en el territorio regional en las zonas que muestran mayor densidad en la variable de las Unidades Escolares.

Figura 5. Getis-Ord de Centros de Educación en Extremadura

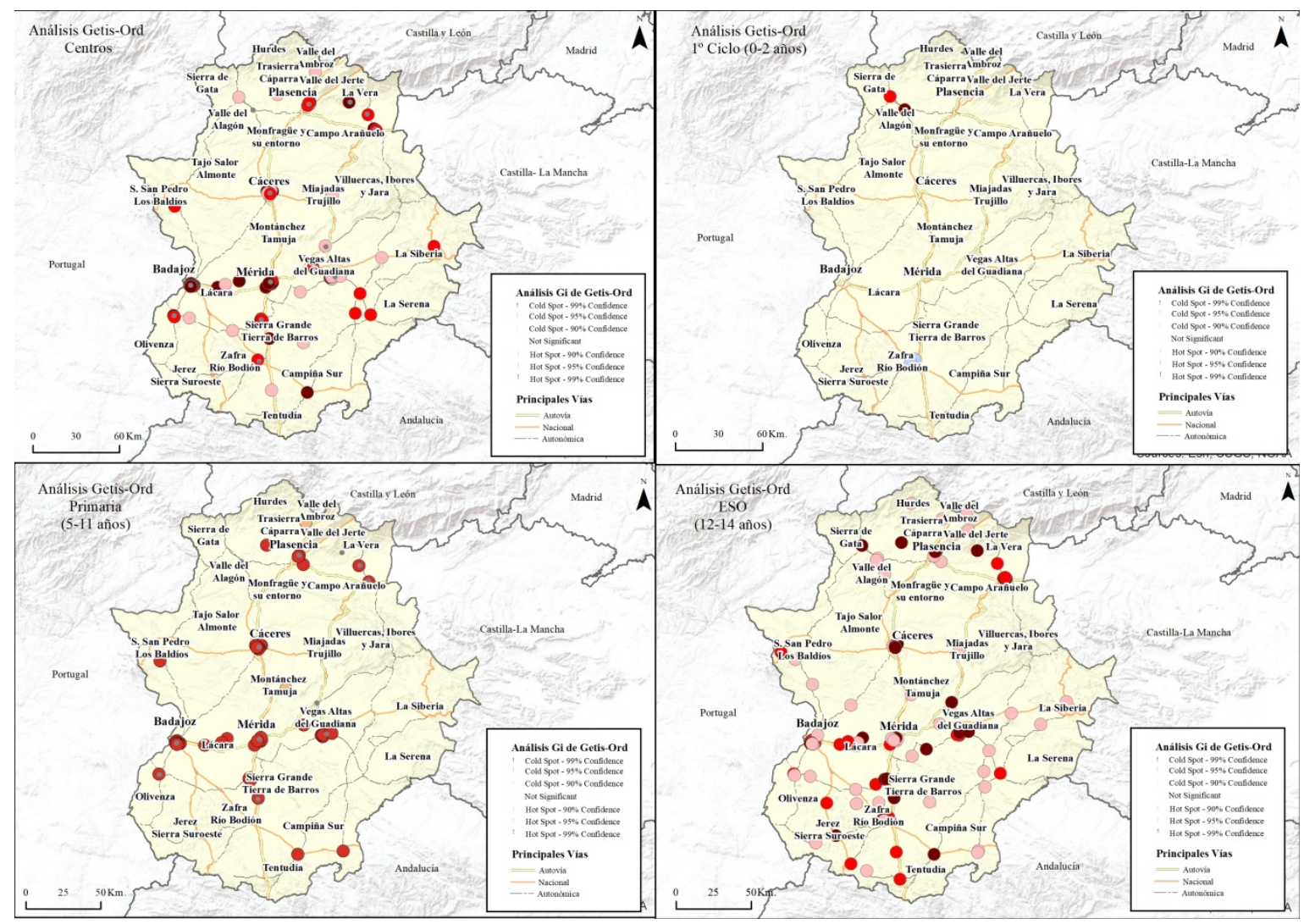

Fuente: elaboración propia a partir de los INE y de la Junta de Extremadura (2015)

En cuanto a los diferentes recursos educativos, la concentración de unidades significativas se obtienen mayores resultados en las Unidades Escolares de Secundaria que de Primaria. En 97 
secciones censales se concentran el mayor porcentaje de unidades de Secundaria respectivas a la media con valores de Hot Spot entre un 90 y un $99 \%$ de significancia y localizadas en las secciones censales de los núcleos urbanos y en las cabeceras comarcales de las áreas de Sierra de Gata, Valle del Alagón, La Vera, Campo Arañuelo-Jara-lbores, Vegas del Guadiana, Barros OesteSierra Grande, Zafra-Río Bodión, Jerez-Sierra Suroeste y Tentudía. Mientras que en el análisis de las unidades de Primaria sólo encontramos concentración de Unidades en 48 Hot Spot. Se sigue obteniendo los mismos resultados que en análisis anteriores, existen mayores desigualdades en la oferta de Secundaria que en la Primaria porque estos últimos presentan mayor número de centros y unidades escolares en edades tempranas y mejor distribuidos por el territorio.

Se complementa y amplia al análisis anterior. Con el estadístico I local de Moran, se localizaban los puntos con mayor concentración de unidades de Extremadura tanto, en el análisis de las Unidades Totales (36 con valores representativos) como en las Unidades de Primaria (35) y de Secundaria (22). Con Getis-Ord nos distingue donde se localizan los puntos de mayor concentración de equipamientos (78, en total de Unidades Escolares, 98 en Secundaria y 48 en Primaria) respecto al promedio en cada caso. Con ambos análisis se han localizado donde existe la mayor concentración de recursos y se ha determinado que en Primaria no existe tantas diferencias entre unos territorios y otros que en la etapa de Secundaria. Se han localizado también algunas zonas rurales donde la oferta educativa todavía existe escasez y que se sitúan en las fronteras de la Comunidad Autónoma de Extremadura y en las zonas de montaña y que serían los territorios donde la Administración debe todavía realizar mejoras en la dotación de sus equipamientos o en el acceso a los mismos. No se han distinguido puntos fríos donde haya concentración de valores bajos.

En ambos análisis los resultados obtenidos en Educación Infantil no son representativos por lo que no existen grandes concentraciones debido a la escasez de plazas públicas que se ofertan en Extremadura. Es un dato que tendría que tener en cuenta también la Administración como una mejora a solventar en los próximos años.

Por último, con el análisis de Densidad Kernel las cantidades conocidas de los elementos puntuales de Unidades Escolares en cada etapa educativa se expanden en el territorio. El estudio de patrón de puntos determinará si existen desequilibrios espaciales en la distribución de este tipo de equipamientos en la región, atendiendo a los principios de calidad de vida, eficiencia y sostenibilidad (Moreno \& Vinuesa, 2009, p. 248) enunciados al inicio de este estudio.

A la luz de los resultados cartográficos sobre densidad de Kernel para los Centros que imparten Educación Infantil de Primer Ciclo (0-3 años) se concentran en los grandes centros de producción y consumo donde la demanda es mayor (Plasencia, Cáceres, Mérida y Badajoz) y los ejes Vegas del Guadiana-Barros en la provincia de Badajoz y el regadío del N. de Cáceres (Campo Arañuelo y Valle del Alagón) (Figura 6). En niveles de Enseñanza Obligatoria, como la etapa de Educación 
Primaria (466 centros), gran parte de este servicio público tiende a ubicarse en los centros neurálgicos de la región, observándose una menor densidad en comarcas como: Villuercas-lboresJara, La Siberia, La Serena, Tentudía, Jerez-Sierra Suroeste, Olivenza, Miajadas- Trujillo, Tajo-SalorAlmonte, Monfragüe o Sierra de San Pedro-Los Baldíos. Esta tendencia es notoria para los Centros de Educación Secundaria (191), pues su menor número favorece la conformación de aglomeraciones en los principales ejes de desarrollo urbano; lo cual determina que existe una movilidad diaria de estudiantes desde los asentamientos rurales a los centros de cabecera comarcal en los que se ubican las instalaciones. Para el nivel de Secundaria, a las comarcas previamente mencionadas por la menor presencia de centros Educación Primaria, debemos añadir las que siguen: Montánchez-Tamuja, Sierra de Gata, Hurdes, Trasierra-Cáparra, Ambroz o Valle del Jerte.

Figura 6. Densidad Kernel de Centros Educativos Extremeños

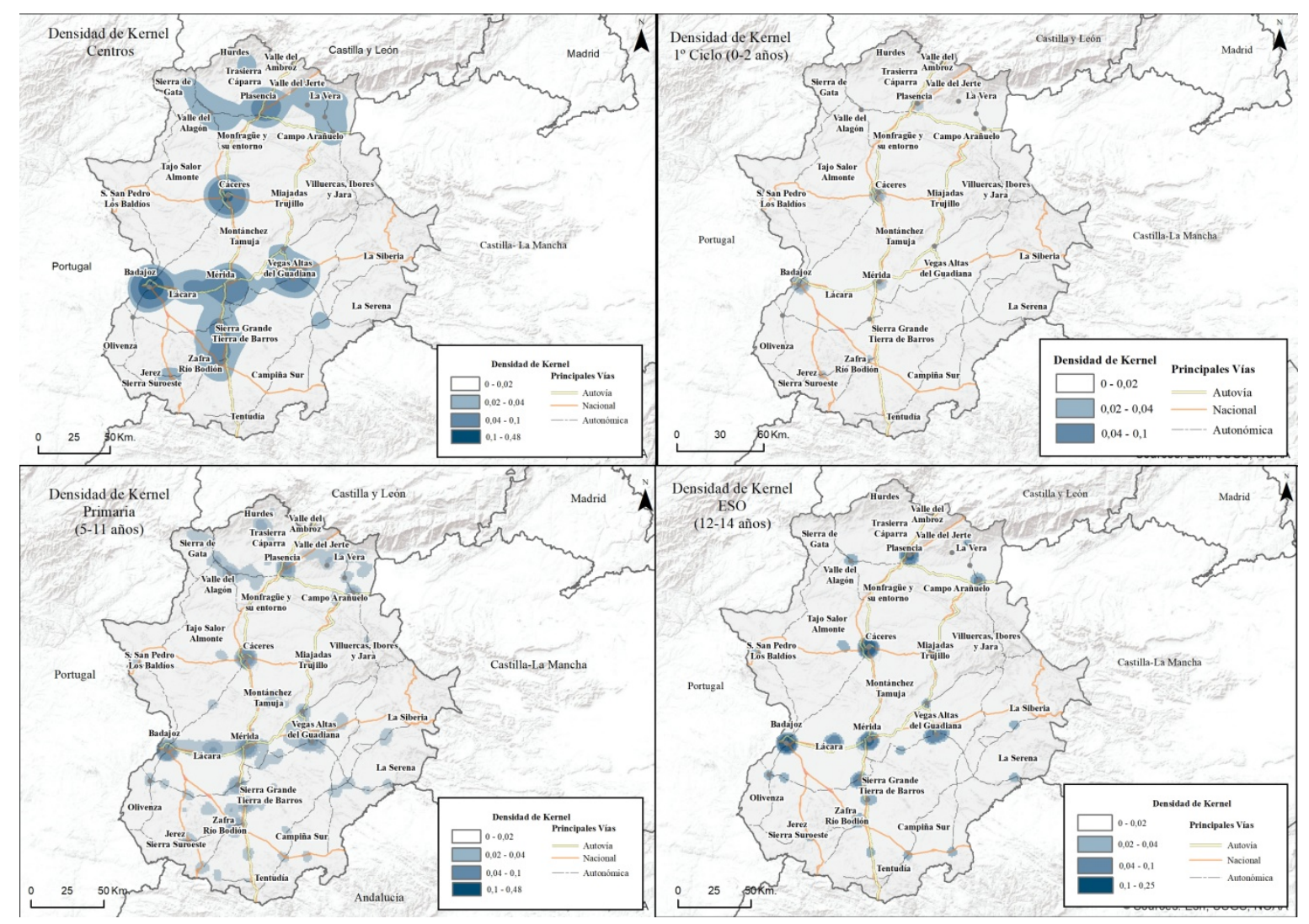

Fuente: elaboración propia a partir de los INE y de la Junta de Extremadura (2015)

Como síntesis de este trabajo, la cartografía de Densidad Kernel nos muestra para las variables analizadas que, un notable número de los Centros de Enseñanza, sobre todo en la Ełapa de Secundaria y en Educación Infantil, tienden a localizarse en las principales urbes de Extremadura y en las franjas este-oeste del regadío en ambas provincias, en respuesta a su estructura poblacional, económica, de infraestructuras y servicios. 


\section{Conclusiones}

A pesar de su importante legado histórico, tanto natural como cultural, Extremadura cuenta con unas características poblacionales, territoriales y económicas que la han situado con un notable retraso socioeconómico respecto a otras regiones de España o Europa. Presenta un generalizado envejecimiento poblacional a excepción de los centros neurálgicos próximos a los principales ejes de comunicación (Autovías a Madrid y Ruta de la Plata) como se ha comprobado con la localización espacial del Índice de Juventud, que actúan como polos de atracción acogiendo gran parte de los equipamientos y servicios, entre ellos los que se ha analizado en este trabajo y que son fundamentales para el mantenimiento de la población, los educativos.

Por ello, desde las Administraciones Públicas se ha realizado una importante labor social, con la implantación y diseño de la red educativa para intentar paliar desequilibrios entre núcleos urbanos y rurales y la mejora del mismo con la construcción de nuevos Centros y dotación de más unidades. También se pretende a la vez, lograr el mantenimiento de una población joven en los espacios más rurales y asentar nuevos yacimientos de empleo.

Con este trabajo se ha analizado la distribución territorial de estos servicios educativos, para determinar si existen áreas con desequilibrios y si la dotación de unidades escolares está relacionada con la demanda de la población (se ha empleado la población menor de 16 años) o la prioridad de la Administración ha sido conseguir el mismo acceso de todos los extremeños desde cualquier punto del territorio, aunque sean áreas con menor población joven y donde los Centros Educativos tendrán menor números de alumnos por aulas. Se ha realizado también por Ełapas Educativas de la Enseñanza Obligatoria: Educación Infantil de Primer Ciclo (0-3 años), Educación Primaria (5-12 años) y Educación Secundaria Obligatoria (12-16 años) para también comprobar si existen divergencias en las mismas. Se han utilizado cuatro análisis espaciales: Accesibilidad al recurso, con el cálculo del tiempo mínimo al centro más próximo; localización de clústeres o agrupaciones de puntos con valores significativos de unidades con los estadísticos I Anselin Local de Moran y Getis-Ord y por último; determinar las áreas de concentración de recursos con el método de densidad Kernel. La escala de este estudio han sido las 970 secciones censales extremeñas y su representación espacial han sido los puntos centrales de los núcleos de población de dichas secciones. A esta capa puntual se le ha añadido la oferta de unidades de cada etapa escolar localizada en su extensión superficial.

Estas diferentes metodologías nos han permitido obtener resultados de gran relevancia territorial. Se distingue una concentración de servicios educativos en ciudades con incipientes dinámicas sociodemográficas próximas a los principales ejes de comunicación, aunque los resultados cartográficos nos muestran una oferta de servicios educativos en edades tempranas en prácticamente todo el territorio de Extremadura, sin apreciarse áreas con manifiesto desequilibrio de 
estos equipamientos (se localizan estos recursos con un alto volumen de Unidades Educativas aunque presenten un indicador de juventud inferior al $15 \%$ ). Por ello, se puede afirmar que en la planificación educativa extremeña ha primado el elemento de proximidad y accesibilidad de la población a los centros de enseñanza sobre el factor de demanda potencial de jóvenes menores de 16 años. Asimismo, para afianzar esta conclusión se ha aplicado la técnica estadística de correlación de Pearson para estimar el nivel de dependencia entre las variables de Unidades totales y la población joven (menores de 16 años) y la correlación obtenida entre ambas entidades no es representativa, pues en la definición del número de plazas ofertadas no se ha considerado el volumen de población demandante sino más bien la accesibilidad geográfica.

Todos estos análisis nos han proporcionado una amplia visión de la distribución de los recursos educativos en la región extremeña. Para la Administración su principal prioridad ha sido establecer una red de Centros de Educación Primaria debido a que afecta a una población de edades tempranas y que puede ser la base para el mantenimiento de población joven (sus progenitores) en los espacios más ruralizados. La Enseñanza Secundaria Obligatoria también se ha ampliado pero se apoya también en el transporte escolar en determinadas áreas muy ruralizadas (jóvenes de 12 a 16 años se desplazan diariamente a centros situados en su cabecera comarcal o núcleo más próximos en transporte escolar, aunque nunca superándose más de 20-25 minutos de desplazamiento). Aunque se aprecia una tendencia a una leve concentración de recursos en las áreas más dinámicas de la región (los puntos calientes o áreas de mayor Densidad de Kernel) pero sin ser excesivamente pronunciada con el resto de la región.

A modo de reflexión final, la elaboración de políticas públicas de desarrollo territorial precisa del conocimiento de las teorías de localización y de un diagnóstico de la situación real del espacio de estudio para tratar de conciliar los principios de equidad, eficiencia y justicia espacial en lo que respecta a la localización de los recursos colectivos. Los Sistemas de Información Geográfica constituyen una herramienta de gran relevancia para el proceso de la gestión del territorio permitiendo detectar las áreas que presentan serias dificultades de acceso a los equipamientos de gestión pública o privada. En suma, los resultados obtenidos pretenden ser una invitación a discutir e identificar alguna de las líneas futuras de trabajo: determinar la localización idónea de nuevos Centros en espacios extremeños que presenten deficiencias, teniendo en cuenta su contexto social y demográfico; definir propuestas de actuación para mejorar las prestaciones educativas con el objetivo de proporcionar a todos los ciudadanos una escuela de calidad al margen de su contexto, etc. Todas estas políticas de mejora de las condiciones de vida y de recursos en los espacios rurales se pueden considerar que están resultando insuficientes en Extremadura, debido a que siguen perdiendo población y sobre todo de efectivos jóvenes que emigran a otras zonas, como se ha comprobado en la localización espacial del Índice de Juventud. Se tiene que seguir 
ampliando y mejorando el diseño de las mismas para intentar paralizar el abandono de estos espacios.

Con este trabajo se plantea también la necesidad de seguir avanzando en otras líneas futuras para comprobar si la oferta de plazas escolares está siendo infrautilizadas y si en el futuro se podrán mantener. La red de centros educativos extremeños, sobre todo en Primaria, se ha diseñado, atendiendo a la accesibilidad para intentar que no haya discriminación social entre unos espacios y otros pero debemos plantearnos si esta oferta es excesiva para la población joven que habita en estos espacios y si en el futuro (en los próximos 15-20 años) con la tendencias bajas de natalidad se podrán seguir manteniendo. Por ello, en los siguientes estudios se pretenden ampliar estos análisis utilizando las mismas metodologías pero incluyendo como variables de estudio el índice de ocupación de estas unidades escolares. Así, como analizar no sólo la oferta de Unidades Escolares sino la calidad y diversidad de la misma, incluyendo la oferta de actividades complementarias, itinerarios formativos o ayudas a la diversidad, por si estas variables condicionan todavía más las diferencias entre espacios urbanos y rurales.

Declaración responsable: Las autoras declaran que no existe ningún conflicto de interés en relación a la publicación de este artículo. Los datos educativos y demográficos (los educativos facilitados por la Consejería de Educación de la Junta de Extremadura) fueron previamente analizados por la Dra. Ana Nieto Masot y codificados en una base de datos para posteriormente poder introducirse en un Sistema de Información Geográfica. La Investigadora Nieves Márquez elaboró y corrigió la capa de datos puntuales de las secciones censales. La metodología del trabajo fue diseñada por la Dra. Ana Nieto Masot. Las dos autoras han colaborado posteriormente en la realización de los análisis de redes y distintos análisis geoestadísticos en el software ArcGis de los datos educativos de la región extremeña. También han participado ambas en la redacción del texto y en la revisión bibliográfica. 


\section{Bibliografía}

Anselin, L. (1995). Local Indicators of Spatial Association-LISA. Geographical Analysis, 27(2), 93115.

Calvo, J. L., Pueyo, A., Jover, J. M., \& Erdozain, O. (2001). Análisis, diagnóstico y ordenación de equipamientos mediante formulaciones cartografiables: valoración de la accesibilidad y requerimientos de la asistencia hospitalaria en la CC. AA. de La Rioja mediante la técnica de potencialidades. Berceo, 141, 247-268.

Carles, J., \& Hoberg, K. (2008). Nuevas técnicas de estadística espacial para la detección de clusters residenciales de población inmigrante. Scripta Nova, XII(263). Retrieved from http://www.ub.edu/geocrit/sn/sn-263.htm

Celemín, J. P. (2009). Autocorrelación espacial e indicadores locales de asociación espacial. Importancia, estructura y aplicación. Revista Universitaria de Geografía, 18, 11-31.

De la Fuente, H. E., Rojas, C., \& Salado, M. J. (2013). Distribución de los equipamientos educativos. Evidencias de inequidad espacial en la educación del área metropolitana de Concepción. GeoFocus, 13(2), 231-257.

De Cos Guerra, O. (2004). Valoración del método de densidades focales (kernel) para la identificación de los patrones espaciales de crecimiento de la población en España. GeoFocus, 4, $136-165$.

Escalona, A. I., \& Díez, C. (2003). Accesibilidad geográfica de la población rural a los servicios básicos de salud: estudio en la provincia de Teruel. AGER, 3, 111-149.

Escribano, J. (2005). La distribución espacial de los equipamientos geriátricos en la comarca de La Ribera Baixa: un análisis prospectivo par la satisfacción de su demanda. Cuadernos de Geografía, 77.

Escribano, J. (2011). El valor de los servicios educativos y sanitarios en los procesos de atracción y mantenimiento de población en medio rural. Revista de Estudios sobre Despoblación y Desarrollo Rural, 13, 11-51.

Galacho, F. B., \& Reyes, S. (2015). Estimación de pautas de asociación y patrones de distribución de edificaciones aisladas en espacios rurales mediante SIG y técnicas basadas en procesos puntuales. In De la Riva, J., P. Ibarra, R. Montorio, \& R. Rodrigues (Eds.), Actas del XXIV Congreso de la Asociación de Geógrafos Españoles (pp. 601-610). Zaragoza: Asociación de Geógrafos Españoles.

Getis, A., \& Ord, J. K. (1992). The Analysis of Spatial Association by Use of Distance Statistics. Geographical Analysis, 4(3), 189-206. 
González, M. A. (2007). Análisis de datos multivariantes: inferencia en poblaciones normales con una y dos muestras. La Laguna: Servicio de Publicaciones, Universidad de La Laguna.

Gutiérrez, J., Cristóbal, C., \& Gómez, G. (2000). Accesibilidad peatonal a la red de metro de Madrid: efectos del Plan de Ampliación 1995-99. Anales de Geografía de la Universidad Complutense, 20, 451-464.

Gutiérrez, J., García, J. C., Alventosa, C., Redondo, J. C., \& Paniagua, E. (2002). Accesibilidad peatonal a la red sanitaria de asistencia primaria en Madrid. Anales de Geografía de la Universidad Complutense, vol. extraordinario, 269-280.

Flores, V., \& Rojas, C. (2009). Localización de instalaciones no deseables: aportes desde la Geografía a la instalación de rellenos sanitarios en el área metropolitana de Concepción. Sociedad Chilena de las Ciencias Geográficas, 9, 298-305.

Fuenzalida, M., \& Moreno, A. (2010). Diseño con SIG de la localización óptima de centros de atención primaria de salud, discriminando según estatus socioeconómico. In J. Ojeda, M. Pita, \& F. Vallejo (Eds.), Tecnologías de la Información Geográfica: La Información Geográfica al servicio de los ciudadanos (pp. 453-465). Sevilla: Secretariado de Publicaciones de la Universidad de Sevilla.

Hair, J. (2008). Análisis multivariante. Madrid: Prentice Hall.

Harvey, D. (1992). Urbanismo y desigualdad social. Madrid: Siglo XXI.

Izquierdo, J. (2002). Manual para agentes de desarrollo rural: ideas y propuestas para moverse entre la conservación del patrimonio y el desarrollo local. Madrid: Mundi-Prensa.

Iris, P. (2015). Los métodos de autocorrelación espacial para la regionalización intraurbana. In G. Buzai (Coord.), Teoría y métodos de la Geografía cuantitativa. Libro 1: Por una Geografía de lo real (pp. 139-154), Buenos Aires: MCA Libros.

Linares, S. (2008). Análisis de la accesibilidad diferencial a equipamientos colectivos mediante SIG. El caso de la ciudad de Tandil. Paper presented at the X Jornadas Cuyanas de Geografía: La Geografía frente a la necesidad de integrar territorios y voluntades, Mendoza.

Martínez, C. (2013). Evaluación de programas. Modelos y procedimientos. Madrid: UNED.

Moreno, A. (2003). Evaluación de la accesibilidad a los equipamientos y determinación de áreas de servicio. In C. Ordoñez (Coord.), Sistemas de información geográfica: Aplicaciones prácticas con IDRISI32 al análisis de riesgos naturales y problemáticas medioambientales (pp. 219-245). Madrid: Ra-Ma. 
Moreno, A. (2004). Modelos de localización óptima de instalaciones y equipamientos. In J. Bosque, \& A. Moreno, SIG y localización óptima de instalaciones y equipamientos (pp. 73-122). Madrid, Ra-MA.

Nieto, A., \& Cárdenas, G. (2015a). Research on the accessibility to health and educational services in the rural areas of Extremadura. European Countryside, 7, 57-67.

Nieto, A., \& Cárdenas, G. (2015b). El Método Leader como política de desarrollo rural en Extremadura en los últimos 20 años (1991-2013). Boletín de la Asociación de Geógrafos Españoles, 69, 139-162.

Oberti, M. (2007). Social and school differentiation in urban space: inequalities and local configurations. Environment and Planning A, 39, 208-227.

Palomar, M. P., \& Bosque, J. (2004) Diagnóstico de la relación entre oferta y demanda potencial de educación primaria en el área metropolitana de Guadalajara, México. Un análisis utilizando el programa LOCALIZA. In C. Conesa, Y. Rogel, \& C. Granell (Eds.), El empleo de los SIG y la Teledetección en Planificación Territorial (pp. 485-496). Murcia: Servicio de Publicaciones de la Universidad de Murcia.

Pearson, K. (1896). Contributions to the mathematical theory of evolution, III: regression. Heredity and panmixia. Philosophical Transactions of the Royal Society of London, 187, 253-318.

Pitarch, M. D. (2000). Los modelos de planificación espacial de los servicios públicos: El caso de los servicios educativos. Cuadernos de Geografía, 67-68, 119-136.

Prat, E., Sánchez, J., Pesquer, L., Olivet, M., Aloy, J., Fusté, J., \& Pons, X. (2008). Estudio sobre la accesibilidad de los centros sanitarios públicos de Cataluña. In L. Hernández, \& J. M. Parreño (Eds.), Tecnologías de la Información (pp. 396-411). Palmas de Gran Canaria: Servicio de Publicaciones y Difusión Científica de la ULPGC.

Pueyo, A. (1991). El sistema de Información Geográfica: un instrumento para la planificación y gestión urbana. Geographicalia, 28, 175-192.

Ramírez, M. L. (2007). Estudio de la accesibilidad de la población del Gran Resistencia a los centros de salud. Paper presented at the XI Conferencia Iberoamericana de Sistemas de Información Geográfica (XI CONFIBSIG), Buenos Aires.

Reynaud, A. (1982). Société, espace et justice. Revue de géographie de Lyon, 57(4), 425. Retrieved from https://www.persee.fr/doc/geoca_0035-113x_1982_num_57_4_6175

Rivera, Y. (2014). Bases de datos geográficas y Autocorrelación espacial para identificar patrones de distribuciones espaciales. Revista Investigación e Innovación en Ingenierías, 2, 18-25. 
Rodríguez, V. (2010). Medición de la accesibilidad geográfica de la población a la red de hospitales de alta resolución de Andalucía mediante Sistemas de Información Geográfica. In J. Ojeda, M. F. Pita, \& I. Vallejo (Eds.), Tecnologías de la Información Geográfica: La Información Geográfica al servicio de los ciudadanos (pp. 549-564). Sevilla: Secretariado de Publicaciones de la Universidad de Sevilla.

Rodríguez, V. (2011). Medición de la accesibilidad geográfica de la población a los Hospitales de Alta Resolución de Andalucía mediante herramientas SIG basadas en el análisis de redes. GeoFocus, 11, 265-292.

Sabuda, F., Ares, S., \& Mikkelsen, C. (2005). Calidad de vida y accesibilidad geográfica en la ciudad de Mar del Plata, primeros aportes. Paper presented at the $\mathrm{X}$ Jornadas Argentinas de Estudios de Población, Mar del Plata, Argentina.

Talen, E. (2001). School, community and spatial equity: An empirical investigation of access to elementary schools in West Virginia. Annals of the Association of American Geographers, 91(3), $465-486$.

Vilalta y Perdomo, C. J. (2005). Cómo enseñar autocorrelación espacial. Economía, Sociedad y Territorio, V(18), 323-333.

Villanueba, A. (2010). Accesibilidad geográfica a los sistemas de salud y educación. Análisis espacial de las localidades de Necochea y Quequén. Revista Transporte y Territorio, 2, 136-157. 